\title{
A Phase I Study of Hypofractionated Carbon-ion Radiotherapy for Stage III Non-small Cell Lung Cancer
}

\author{
JUN-ICHI SAITOH ${ }^{1}$, KATSUYUKI SHIRAI ${ }^{1}$, TAKANORI ABE ${ }^{1}$, NOBUTERU KUBO ${ }^{1}$, \\ TAKESHI EBARA ${ }^{2}$, TATSUYA OHNO $^{1}$, KOICHI MINATO $^{3}$, RYUSEI SAITO $^{4}$, MASANOBU YAMADA $^{5}$, \\ TAKASHI NAKANO ${ }^{1}$ and THE WORKING GROUP OF THE LUNG TUMOR \\ ${ }^{1}$ Gunma University Heavy Ion Medical Center, Gunma, Japan; \\ ${ }^{2}$ Department of Radiation Oncology, Gunma Prefectural Cancer Center, Ota, Japan; \\ ${ }^{3}$ Department of Respiratory Medicine, Gunma Prefectural Cancer Center, Ota, Japan; \\ ${ }^{4}$ Department of Respiratory Medicine, National Hospital Organization Shibukawa Medical Center, Shibukawa, Japan; \\ ${ }^{5}$ Department of Medicine and Molecular Science, Gunma University Graduate School of Medicine, Maebashi, Japan
}

\begin{abstract}
Background/Aim: The aim of this study was to assess the feasibility and safety of hypofractionated carbonion radiotherapy $(C$-ion $R T)$ in patients with stage III nonsmall cell lung cancer (NSCLC). Patients and Methods: Patients with untreated, histologically proven, unresectable stage III NSCLC and not candidates for chemotherapy were included in this study. C-ion RT was planned and administered with 4 Gy (relative biological effectiveness $(R B E))$ in daily fractions for a total dose of $64 G y(R B E)$ without combined chemotherapy. Dose-limiting toxicity (DLT) was defined as suspension of C-ion RT treatment for 2 weeks due to $\geq$ grade 2 pneumonitis, or any other $\geq$ grade 3 adverse event, or as any $\geq$ grade 4 adverse event within 3 months from the start of treatment. Results: Six patients were treated between June 2013 and December 2014. The planned full dose of C-ion RT (64 Gy (RBE)) was completed in all patients. No patient developed DLT, and no patient experienced toxicities of $\geq$ grade 3 severity. The overall response rate was 100\%, and local tumor control was achieved in all patients during the survival period. Conclusion: Hypofractionated C-ion RT of patients with stage III NSCLC was feasible and well tolerated. Although the number of patients in this study was small, the results support further investigations to confirm the long-term therapeutic efficacy of this treatment.
\end{abstract}

Correspondence to: Jun-ichi Saitoh, Gunma University Heavy Ion Medical Center, 3-39-22, Showa-machi, Maebashi, Gunma 3718511, Japan. Tel: +81 272208383, Fax: +81 272208397, e-mail: junsaito@gunma-u.ac.jp

Key Words: Stage III non-small cell lung cancer, carbon-ion radiotherapy, phase $\mathrm{I}$.
Stage III locally advanced non-small cell lung cancer (NSCLC) accounts for approximately $25 \%$ of all lung cancer cases (1). At present, concurrent use of platinum-based chemotherapy with thoracic radiotherapy is the standard treatment for inoperable stage III NSCLC. The median survival time for stage III NSCLC treated with concurrent chemoradiotherapy (CCRT) was 20 to 27 months, and the local recurrence rate was reported to be $40-60 \%$ (2-4). The treatment outcomes of CCRT for stage III NSCLC are still unsatisfactory.

CCRT is not strongly recommended for elderly patients or patients with poor performance status. A population-based analysis of radiation therapy alone for elderly patients with unresected stage III NSCLC was reported in 2013; the median overall survival (OS) for 6,468 patients was 9.0 months (5). Treatment failure was more frequent in patients treated by radiotherapy alone than in those treated by CCRT. To intensify the treatment, hypofractionated radiotherapy by $\mathrm{X}$-ray radiotherapy and proton beam radiotherapy was proposed (6-8).

Compared to photons, carbon ions have higher linear energy transfer and larger relative biological effectiveness (RBE), and therefore offer a higher probability of tumor control $(9,10)$. The physical characteristics of carbon ions, such as the Bragg peak and small lateral scattering, are theoretically superior to those of photons, in that carbon ions can allow a more localized delivery of the radiation dose. Hypofractionated schedules of carbon-ion radiotherapy (C-ion RT) have been adopted for many sites of malignancies. In patients with stage I lung cancer treated with $\mathrm{C}$-ion $\mathrm{RT}$ in four fractions, the 5-year local control rate was $95 \%$ for $\mathrm{T} 1$ tumors and $80 \%$ for T2 tumors (11). A comparison of the dose-volume histograms of C-ion RT with those of stereotactic body X-ray radiotherapy in peripheral stage I 
Table I. Planned dose levels.

\begin{tabular}{llcc}
\hline Level & PTV1 & PTV2 & PTV3 \\
\hline 0 & 40 Gy (RBE) in 10 fractions & 12 Gy (RBE) in 3 fractions & 8 Gy (RBE) in 2 fractions \\
1 & 40 Gy (RBE) in 10 fractions & 12 Gy (RBE) in 3 fractions & 12 Gy (RBE) in 3 fractions \\
\hline
\end{tabular}

PTV: Planning target volume; RBE: relative biological effectiveness.

lung cancer showed that the dose distribution of C-ion RT had better target conformity and spared normal tissues of the lung, spinal cord, heart, esophagus, and trachea (12).

The techniques of hilar and mediastinal irradiation by $\mathrm{C}$ ion RT have not been completely established. In a preliminary examination, we compared treatment planning by photon radiotherapy with planning by C-ion RT for patients with stage III NSCLC. C-ion RT irradiated targets with greater homogeneity and with lower doses to organs at risk (OARs), such as the lungs, spinal cord, esophagus, bone, and trachea, than did X-ray radiotherapy (13). Therefore, we conducted a prospective phase I study of hypofractionated C-ion RT for the treatment of stage III NSCLC.

\section{Patients and Methods}

Study design. To determine the dose and fractionation schedule in Cion RT, more than 50 phase I/II dose escalation studies at various sites of malignancies were performed at the National Institute of Radiological Sciences Hospital (NIRS) in Chiba (14). The optimal treatment schedule was determined as 64 Gy (RBE) in 16 fractions with 4 fractions per week for the treatment of head and neck cancer, and the 5-year local control rate ranged from $61 \%$ to $75 \%$ (15). Tolerable doses for the esophagus and respiratory tract have not yet been fully investigated. For treatment of solitary lymph node metastasis, C-ion RT of 52.8 Gy (RBE) in 12 fractions with 4 fractions per week is sometimes performed, and the efficacy and safety of this treatment have been confirmed at NIRS and our facility. Based on these results, we targeted the dose and fractionation schedule as $64 \mathrm{~Gy}$ (RBE) in 16 fractions for primary tumors and metastatic lymph nodes that are not close to OARs and as 52 Gy (RBE) in 12 fractions for targets that are close to OARs. The primary endpoint was defined as the rate of occurrence of acute adverse events. The secondary endpoints were defined as the response rate, local control rate, failure pattern, and occurrence of late adverse events.

Patients. The eligibility criteria for patients were as follows: histologically proven unresectable stage III NSCLC; no previous chemotherapy or radiotherapy; a performance status of $0-2$ on the Eastern Cooperative Oncology Group scale; a life expectancy of 6 months or more; ineligible for chemotherapy. Patients were excluded if they had any of the following: malignant pericardial or pleural effusions; tumor invasion to heart, large vessel, trachea or esophagus; active double cancer; interstitial pneumonitis; intractable infectious disease in the irradiated area; tumor uncovered by a field size of $15 \times 15 \mathrm{~cm}$; N3 disease; pregnancy; or breast-feeding.
The study was approved by the institutional ethics committee, and written informed consent was obtained from all patients. The study is registered with the University Hospital Medical Information Network in Japan (UMIN 000011041).

Treatment planning. The patient was immobilized in the supine or prone position using a thermoplastic shell (Shellfitter; Sanyo Polymer Industrial, Nara, Japan) and a pillow made of watersclerogenic polymers (Moldcare; ALCARE, Tokyo, Japan). The patient was turned around the superior-inferior axis to achieve adequate posture if oblique beam irradiation was needed, and computed tomography (CT) images were obtained in 2-mm-thick slices. First, a respiratory-gated CT image was acquired around the end of expiration. Then a four-dimensional CT scan was acquired to quantify respiratory motion. In actual treatment, the gating level for respiratory-gated irradiation was within $30 \%$ of the wave height around peak exhalation. The gross tumor volume (GTV) included the primary gross tumor and lymph nodes over $1 \mathrm{~cm}$ in short axis diameter. The clinical target volume (CTV) included the GTV and prophylactic lymph nodal areas of the ipsilateral hilar and mediastinal lymph nodes. Planning target volume 1 (PTV1) was defined as the CTV plus a 5- to $10-\mathrm{mm}$ margin in all directions. PTV was phased down in the latter half of the treatment. PTV2 did not include prophylactic lymph nodal areas. PTV2 was defined as the GTV plus a 5- to $10-\mathrm{mm}$ margin in all directions. When the GTV was close to OARs such as the esophagus or respiratory tract, PTV3, which excludes OARs, was used. The clinical dose distribution was calculated from the physical dose and RBE based on the experimental results (9). The unit of clinical dose was described as "Gy (RBE)." Passive scattering carbon ion dose distribution was calculated by XiO-N (ELEKTA and Mitsubishi Electric, Tokyo, Japan). The dose was administered to the isocenter of the PTV, and the fractional dose was 4 Gy (RBE). The planned dose levels are shown in Table I. For dose level 1 setting, PTV1 received a total dose of $40 \mathrm{~Gy}(\mathrm{RBE})$ in 10 fractions. PTV2 received an additional dose of 12 Gy (RBE) in three fractions. PTV3 received an additional dose of $12 \mathrm{~Gy}$ (RBE) in three fractions, and the total dose for PTV3 was 64 Gy (RBE). The maximum dose to the OARs was defined as follows: spinal cord $30 \mathrm{~Gy}$ (RBE), esophagus $60 \mathrm{~Gy}$ (RBE), trachea and main bronchus $60 \mathrm{~Gy}$ (RBE), stomach or bowel 40 Gy (RBE), and brachial plexus 60 Gy (RBE). The doses were de-escalated to level 0 (the total dose for PTV3 of $60 \mathrm{~Gy}(\mathrm{RBE})$ ) according to the frequency of dose-limiting toxicity (DLT). Toxicities were assessed by the Common Terminology Criteria for Adverse Events, version 4.0. DLT was defined as suspension of C-ion RT treatment for 2 weeks due to $\geq$ grade 2 pneumonitis or other $\geq$ grade 3 adverse event during treatment, and any $\geq$ grade 4 adverse event within 3 months from the start of treatment. 


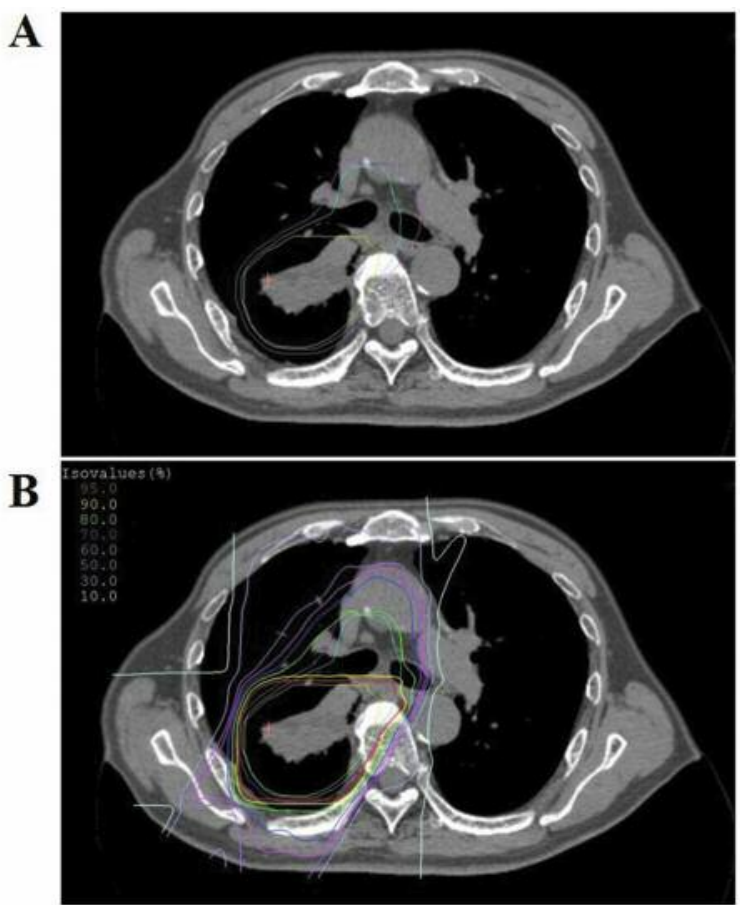

Figure 1. Delineation of planning target volume (PTV) and dose distribution of carbon-ion radiotherapy. (A) Definition of PTV. PTV1, PTV2, PTV3 was contoured with a magenta line, a cyan line, and a yellow line, respectively. (B) Dose distribution in axial view.

At least six patients were enrolled at each dose level. Initially, six patients were treated at dose level 1 . If two of the six patients had a DLT at level 1 , dose reduction to level 0 was planned. The delineation of the PTVs and dose distribution of a representative case with axial images is shown in Figure 1.

Treatment evaluation. Prior to treatment, the patients were evaluated by whole blood cell count, differential count, routine chemistry measurements, electrocardiogram, pulmonary function tests, chest radiography, chest $\mathrm{CT}$, abdominal $\mathrm{CT}$, whole-brain magnetic resonance imaging or $\mathrm{CT}$, and ${ }^{18} \mathrm{~F}$-fluorodeoxyglucose positron emission tomography. During treatment, chest X-ray, complete blood cell count, differential count, routine chemistry measurements, physical examination, and toxicity assessments were performed at least once a week. Chest CT was performed as needed for replanning of C-ion RT depending on tumor shrinkage. Chest CT was also performed at the end of treatment and every 3 months after the start of C-ion RT. Tumor response was evaluated according to Response Evaluation Criteria in Solid Tumors (RECIST) version 1.1 (16).

Statistical analysis. Statistical analysis was performed with IBM SPSS Statistics 21.0 software (IBM, Armonk, NY, USA). Survival time was measured from the first day of treatment to the date of death or last follow-up. The Kaplan-Meier method was employed to determine the actuarial survival rate.

Ethics approval and consent to participate. The study was approved by the institutional ethics committee, and written informed consent
Table II. Patient characteristics.

\begin{tabular}{ccccccc}
\hline $\begin{array}{c}\text { Case } \\
\text { Age } \\
(\mathrm{y})\end{array}$ & Gender & Histology & PS & Stage & $\begin{array}{c}\text { Brinkman } \\
\text { index }\end{array}$ \\
\hline 1 & 78 & Male & Sq & 1 & T2bN2M0 & 2280 \\
2 & 76 & Female & Ad & 1 & T2bN2M0 & 0 \\
3 & 79 & Male & Sq & 1 & T2aN2M0 & 1800 \\
4 & 64 & Male & Ad & 1 & T3N2M0 & 800 \\
5 & 70 & Female & Sq & 1 & T2aN2M0 & 450 \\
6 & 80 & Female & Ad & 1 & T1bN2M0 & 0 \\
\hline
\end{tabular}

Ad: Adenocarcinoma; PS: performance status; Sq: squamous cell carcinoma.

was obtained from all patients. The study is registered with the University Hospital Medical Information Network in Japan (UMIN 000011041). Registered 25 June 2013, prospectively registered.

URL: https://upload.umin.ac.jp/cgi-open-bin/ctr_e/ctr_view.cgi? recptno=R000012923.

\section{Results}

Patients. Six patients were enrolled at a dose level 1 from June 2013 to December 2014. The median follow-up period was 26 months (range $=4-43$ months). Patient characteristics are listed in Table II. The median age was 77 years, ranging from 64 to 80 years. Three patients were male and three were female. The histology was adenocarcinoma in three patients and squamous cell carcinoma in three. Four patients had a history of smoking. The planned full dose of C-ion RT was administered to all patients at dose level 1 . Tumor shrinkage or improvement in collapse of the lung during C-ion RT was seen in all patients; adaptive replanning was needed once in three patients and twice in three patients.

Dosimetric analysis. A dosimetric analysis of C-ion RT is shown in Table III. D1cc (minimum dose to maximally irradiated $1 \mathrm{cc}$ ) of the trachea, main bronchus, and esophagus did not exceed 60 Gy (RBE) in any case. D1cc of the spinal cord slightly exceeded $30 \mathrm{~Gy}$ (RBE) in one patient. The dose received by $>95 \%$ of PTV3 (D95) was more than 60 Gy (RBE) in five patients.

Assessment of toxicity. Acute and late adverse events are listed in Table IV. No patients experienced toxicities of $\geq$ grade 3 severity. One patient developed grade 2 esophagitis, and another patient developed grade 2 pneumonitis. No patients developed a DLT at dose level 1.

Response rate and follow-up. The patients' clinical courses are shown in Table V. All patients achieved a partial response (overall response rate, 100\%). No patient had an in-field recurrence, but one had a recurrence outside of and around the PTV, which appeared to be a pulmonary metastasis. 
Table III. Dosimetric analysis of carbon-ion radiotherapy.

\begin{tabular}{|c|c|c|c|c|c|c|c|c|c|}
\hline \multirow[t]{2}{*}{ Case } & \multicolumn{3}{|c|}{ Lung } & \multicolumn{2}{|c|}{ Trachea } & \multicolumn{2}{|c|}{ Right main bronchus } & \multicolumn{2}{|c|}{ Left main bronchus } \\
\hline & V5 (\%) & $\mathrm{V} 10(\%)$ & $\mathrm{V} 20(\%)$ & D0.1cc & $\mathrm{D} 1 \mathrm{cc}$ & $\mathrm{D} 0.1 \mathrm{cc}$ & D1cc & D0.1cc & $\mathrm{D} 1 \mathrm{cc}$ \\
\hline 1 & 29.8 & 26.0 & 21.7 & 42.1 & 41.5 & 41.6 & 41.3 & 63.5 & 58.3 \\
\hline 2 & 38.6 & 35.2 & 28.7 & 51.9 & 51.0 & 52.2 & 51.9 & 52.3 & 50.8 \\
\hline 3 & 34.4 & 31.7 & 25.3 & 52.3 & 52.0 & 59.6 & 54.4 & 56.9 & 52.5 \\
\hline 4 & 24.6 & 19.1 & 15.9 & 49.4 & 43.2 & 47.8 & 43.5 & 54.3 & 53.1 \\
\hline 5 & 37.8 & 35.7 & 24.1 & 53.2 & 52.2 & 53.8 & 52.4 & 52.2 & 50.9 \\
\hline \multirow[t]{3}{*}{6} & 24.8 & 22.1 & 16.9 & 54.4 & 52.5 & 54.5 & 51.9 & 49.6 & 42.3 \\
\hline & \multicolumn{2}{|c|}{ Esophagus } & \multicolumn{2}{|c|}{ Spinal cord } & \multicolumn{2}{|c|}{ Heart } & PTV1 & PTV2 & PTV3 \\
\hline & D0.1cc & $\mathrm{D} 1 \mathrm{cc}$ & D0.1cc & D1cc & V50 (cc) & V60 (cc) & D95 & D95 & D95 \\
\hline 1 & 46.0 & 44.1 & 16.0 & 15.2 & 50 & 28 & 38.7 & 50.6 & 61.1 \\
\hline 2 & 48.8 & 43.6 & 39.9 & 32.9 & 34 & 9.2 & 39.1 & 52.0 & 61.2 \\
\hline 3 & 62.4 & 59.7 & 33.8 & 27.0 & 11 & 5.2 & 49.3 & 51.8 & 63.2 \\
\hline 4 & 53.4 & 53.0 & 7.1 & 6.7 & 0.51 & 0 & 44.4 & 52.0 & 63.6 \\
\hline 5 & NA & NA & 33.0 & 20.3 & 15 & 4.4 & 46.5 & 51.5 & 60.1 \\
\hline 6 & 46.7 & 43.4 & 2.5 & 2.2 & 0 & 0 & 41.4 & 51.7 & 55.2 \\
\hline
\end{tabular}

$\mathrm{V} n$ : Total volume irradiated for $>n$ Gy; $\mathrm{D} n$ cc: minimum dose to maximally irradiated $n$ cc; D95: dose received by $>95 \%$ of PTV; PTV: planning target volume; NA: not available (because of a past history of esophagectomy).

Hematogenous distant metastases were seen in four patients, all of whom died of lung cancer. The mean survival time was 26 months, and actuarial 1-year and 2-year OS rates were $83 \%$ and $50 \%$, and the 1-year and 2-year progression-free survival rates were $33 \%$ and $33 \%$, respectively.

\section{Discussion}

The current standard treatment for stage III unresectable NSCLC is CCRT. However, about half the patients with stage III NSCLC have a local recurrence after CCRT (2-4). A randomized dose escalation study comparing high-dose (74 Gy) CCRT with standard-dose (60 Gy) CCRT was performed (17). Median OS was shorter in the high-dose group than in the standard-dose group (20.3 vs. 28.7 months). There were more treatment-related deaths in the high-dose group than in the standard-dose group (eight $v s$. three). The maximum grade of esophagitis and the heart V5 (the percentage of heart volume receiving $\geq 5 \mathrm{~Gy}$ ) and V30 were significant predictors of mortality. There was no significant difference in improvement in local control between the standard-dose group and the high-dose group (17). Hence, dose escalation is not recommended in CCRT for stage III NSCLC, and a novel radiation therapy is needed. With regard to $\mathrm{C}$-ion RT for locally advanced NSCLC, a prospective, nonrandomized phase I/II study in patients with inoperable stage IIA-IIIA NSCLC was reported in 2015 from NIRS (18). The patients received a total dose of 68 to $76 \mathrm{~Gy}$ (RBE) in 16 fractions without concurrent chemotherapy. The 2-year local control and OS rates in 62 patients were $93.1 \%$ and $51.9 \%$, respectively, and the rate of grade 3 or greater toxicity was $3.2 \%$. However, the patients treated were limited to those with $\mathrm{N} 0, \mathrm{~N} 1$, or single station limited N2 lymph node metastasis, and the methods of treatment of the hilar and mediastinal regions and the dose volume parameters of OARs were not mentioned (18). In our study, C-ion RT with a total dose of 64 Gy (RBE) in 16 fractions was performed as a prospective phase I study for the treatment of stage III NSCLC. No patient developed a DLT, and local control was achieved in all patients. Mediastinal irradiation with $52 \mathrm{~Gy}(\mathrm{RBE})$ in 12 fractions and an additional boost of $12 \mathrm{~Gy}$ (RBE) in 3 fractions to the gross tumor were confirmed to be safe and effective.

With regard to the analysis of the failure pattern, four of the six patients had hematogenous distant metastases. Because these four patients were over 75 years old or having complications, there was no indication of chemotherapy. However, because the rate of distant failure was still high in this group, C-ion RT in combination with chemotherapy for non-aged patients will be studied in the future.

Proton beams also have a Bragg peak, and several studies have shown that proton beam radiotherapy can deliver a high dose to tumors while reducing the dose to surrounding normal organs (19-21). To determine the recommended dose of proton beam therapy, a dose escalation study with proton beam therapy was performed. Harada et al. reported the results of a 
Table IV. Acute and late adverse events.

\begin{tabular}{|c|c|c|c|c|c|c|c|c|c|c|c|c|c|c|}
\hline \multirow[t]{2}{*}{ Case } & \multicolumn{7}{|c|}{ Acute adverse events } & \multicolumn{7}{|c|}{ Late adverse events } \\
\hline & Blood & Lung & $\begin{array}{l}\text { Respiratory } \\
\text { tract }\end{array}$ & Esophagus & Heart & Skin & $\begin{array}{c}\text { Chest } \\
\text { wall }\end{array}$ & Blood & Lung & $\begin{array}{l}\text { Respiratory } \\
\text { tract }\end{array}$ & Esophagus & Heart & Skin & $\begin{array}{l}\text { Chest } \\
\text { wall }\end{array}$ \\
\hline 1 & 1 & 0 & 0 & 2 & 0 & 1 & 0 & 1 & 1 & 0 & 0 & 1 & 1 & 1 \\
\hline 2 & 1 & 2 & 0 & 1 & 0 & 1 & 0 & 0 & 2 & 0 & 0 & 0 & 0 & 0 \\
\hline 3 & 1 & 1 & 0 & 1 & 0 & 1 & 0 & 0 & 1 & 0 & 0 & 0 & 0 & 0 \\
\hline 4 & 0 & 0 & 0 & 1 & 0 & 2 & 0 & 0 & 1 & 0 & 0 & 0 & 0 & 1 \\
\hline 5 & 0 & 1 & 0 & NA & 0 & 1 & 0 & NA & NA & NA & NA & NA & NA & NA \\
\hline 6 & 2 & 0 & 0 & 0 & 0 & 1 & 0 & 0 & 1 & 0 & 0 & 0 & 0 & 1 \\
\hline
\end{tabular}

NA: Not available (because of a past history of esophagectomy and an early tumor progression of lung cancer).

Table V. Patients' clinical courses.

\begin{tabular}{|c|c|c|c|c|c|c|}
\hline Case & Follow-up time (mo) & Course & Local recurrence & Nodal recurrence & Distant metastasis & DLT \\
\hline 1 & 43 & Died of NSCLC & No & No & Lung & None \\
\hline 2 & 21 & Died of NSCLC & No & $\begin{array}{l}\text { Supraclavicular lymph node } \\
\text { (outside PTV) }\end{array}$ & Lung & None \\
\hline 3 & 21 & Died of other disease & No & No & No & None \\
\hline 4 & 32 & Died of NSCLC & No & No & Brain, bone & None \\
\hline 5 & 4 & Died of NSCLC & No & No & Lung, liver, skin & None \\
\hline 6 & 31 & Disease-free & No & No & No & None \\
\hline
\end{tabular}

DLT: Dose-limiting toxicity; NSCLC: non-small cell lung cancer; PTV: planning target volume.

dose escalation study of proton beam therapy with concurrent chemotherapy for stage III NSCLC (22). Two prescribed doses of proton beam therapy of 66 Gy (RBE) in 33 fractions and $74 \mathrm{~Gy}(\mathrm{RBE})$ in 37 fractions were tested. One patient treated with 74 Gy (RBE) developed a grade 3 esophageal fistula, and the recommended dose was determined to be 66 Gy (RBE) (22). Gomez et al. conducted a dose escalation study of hypofractionated proton beam therapy for stage III NSCLC (8). Three dose levels were tested: 45 Gy (RBE) in 3-Gy (RBE) fractions, 52.5 Gy (RBE) in 3.5-Gy (RBE) fractions, and $60 \mathrm{~Gy}(\mathrm{RBE})$ in 4-Gy (RBE) fractions. One patient treated with a total dose of $52.5 \mathrm{~Gy}(\mathrm{RBE})$ in 15 fractions developed a tracheoesophageal fistula (8). In a dose escalation study, it is important to maintain safety. In this study, the dose and fractionation schedule was set at $64 \mathrm{~Gy}(\mathrm{RBE})$ in 16 fractions for the gross tumor and 52 Gy (RBE) in 12 fractions for targets that were close to OARs because effectiveness of 64 Gy (RBE) in 16 fractions and safety in mediastinal irradiation of $52.8 \mathrm{~Gy}(\mathrm{RBE})$ in 12 fractions was confirmed in previous studies and from clinical experience. Consequently, in-field tumor control was achieved in all patients without severe toxic events. To maintain safety of the treatment, patient selection is an also important issue. In the previous two studies of dose escalation of proton beam therapy, some patients with $\mathrm{T} 4$ or $\mathrm{N} 3$ disease were enrolled. In the present study, patients with T4 disease invading the heart, large vessels, esophagus, or trachea were not included to maintain safety. Patients with N3 disease were also excluded, because the irradiated area might become very broad and patients with N3 disease have a high potential for microscopic distant metastases (23-25). Accordingly, no patient with stage IIIB disease was enrolled in this study, and the effectiveness and safety of C-ion RT for stage IIIA NSCLC were confirmed.

Adaptive replanning is one of the most important subjects in high-precision radiation therapy. Especially in particle beam therapy, slight anatomic changes may lead to degradation of the dose distribution because the irradiated area is highly localized, and particle beam ranges are very susceptible to changes in the tissue density of the beam pathway or in target shape. In the previous report of hypofractionated proton beam therapy for stage III NSCLC, the authors stated that adaptive replanning was not compulsory and was not performed in any of the patients because of the short course of the treatment regimen (11). However, in our study, tumor shrinkage or improvement in lung collapse developed rapidly in one or two weeks from 
the start of C-ion RT, and adaptive replanning was performed in all patients. Careful observation and repeated confirmation of dose distribution is essential, even if the treatment is performed on a hypofractionated schedule.

Since April 2016, an integrated treatment schedule using 16 fractions over 4 weeks with a total dose of 64 to 72 Gy (RBE) has been applied in a multicenter prospective registry study of Japanese C-ion RT facilities for the treatment of locally advanced NSCLC. Further improvement in treatment results has to be confirmed by a multicenter prospective registry study of C-ion RT for patients with locally advanced NSCLC.

\section{Conclusion}

C-ion RT for the treatment of stage III NSCLC was confirmed to be feasible in this prospective study. Although the number of patients was small, the results support further clinical evaluations to confirm the therapeutic efficacy of this treatment and to prevent or reduce toxicity.

\section{Conflicts of Interest}

The Authors declare they have no competing interests.

\section{Acknowledgements}

This research did not receive any specific grant from funding agencies in the public, commercial, or not-for-profit sectors. This study was supported by the Research Project of Gunma University Heavy Ion Medical Center.

The Authors would like to thank Enago (www.enago.jp) for the English language review.

\section{References}

1 Mountain CF: Revisions in the international system for staging lung cancer. Chest 111: 1710-1717, 1997.

2 Segawa Y, Kiura K, Takigawa N, Kamei H, Harita S, Hiraki S, Watanabe Y, Sugimoto K, Shibayama T, Yonei T, Ueoka H, Takemoto M, Kanazawa S, Takata I, Nogami N, Hotta K, Hiraki A, Tabata M, Matsuo $\mathrm{K}$ and Tanimoto M: Phase III trial comparing docetaxel and cisplatin combination chemotherapy with mitomycin, vindesine, and cisplatin combination chemotherapy with concurrent thoracic radiotherapy in locally advanced non-small-cell lung cancer: OLCSG 0007. J Clin Oncol 28: 3299-3306, 2010.

3 Saitoh J, Saito Y, Kazumoto T, Kudo S, Yoshida D, Ichikawa A, Sakai H, Kurimoto F, Kato S and Shibuya K: Concurrent chemoradiotherapy followed by consolidation chemotherapy with bi-weekly docetaxel and carboplatin for stage III unresectable, non-small-cell lung cancer: clinical application of a protocol used in a previous phase II study. Int J Radiat Oncol Biol Phys 82: 1791-1796, 2012.

4 Yamamoto N, Nakagawa K, Nishimura Y, Tsujino K, Satouchi M, Kudo S, Hida T, Kawahara M, Takeda K, Katakami N, Sawa T, Yokota S, Seto T, Imamura F, Saka H, Iwamoto Y, Semba H, Chiba Y, Uejima H and Fukuoka M: Phase III study comparing second- and third-generation regimens with concurrent thoracic radiotherapy in patients with unresectable stage III non-smallcell lung cancer: West Japan Thoracic Oncology Group WJTOG0105. J Clin Oncol 28: 3739-3745, 2010.

5 Sigel K, Lurslurchachai L, Bonomi M, Mhango G, Bergamo C, Kale M, Halm E and Wisnivesky J: Effectiveness of radiation therapy alone for elderly patients with unresected stage III nonsmall cell lung cancer. Lung Cancer 82: 266-270, 2013.

6 Amini A, Lin SH, Wei C, Allen P, Cox JD and Komaki R: Accelerated hypofractionated radiation therapy compared to conventionally fractionated radiation therapy for the treatment of inoperable non-small cell lung cancer. Radiat Oncol 7: 33, 2012.

7 Nguyen LN, Komaki R, Allen P, Schea RA and Milas L: Effectiveness of accelerated radiotherapy for patients with inoperable non-small cell lung cancer (NSCLC) and borderline prognostic factors without distant metastasis: a retrospective review. Int J Radiat Oncol Biol Phys 44: 1053-1056, 1999.

8 Gomez DR, Gillin M, Liao Z, Wei C, Lin SH, Swanick C, Alvarado T, Komaki R, Cox JD and Chang JY: Phase 1 study of dose escalation in hypofractionated proton beam therapy for non-small cell lung cancer. Int J Radiat Oncol Biol Phys 86: 665-670, 2013.

9 Kanai T, Endo M, Minohara S, Miyahara N, Koyama-ito H, Tomura H, Matsufuji N, Futami Y, Fukumura A, Hiraoka T, Furusawa Y, Ando K, Suzuki M, Soga F and Kawachi K: Biophysical characteristics of HIMAC clinical irradiation system for heavy ion radiation therapy. Int J Radiat Oncol Biol Phys 44: 201-210, 1999.

10 Schulz-Ertner D and Tsujii H: Particle radiation therapy using proton and heavier ion beams. J Clin Oncol 25: 953-964, 2007.

11 Miyamoto T, Baba M, Sugane T, Nakajima M, Yashiro T, Kagei K, Hirasawa N, Sugawara T, Yamamoto N, Koto M, Ezawa H, Kadono K, Tsujii H, Mizoe JE, Yoshikawa K, Kandatsu S, Fujisawa $\mathrm{T}$ and Working Group for Lung Cancer: Carbon ion radiotherapy for stage I non-small cell lung cancer using a regimen of four fractions during 1 week. J Thorac Oncol 2: 916926, 2007.

12 Ebara T, Shimada H, Kawamura H, Shirai K, Saito J, Kawashima M, Tashiro M, Ohno T, Kanai T and Nakano T: Dosimetric analysis between carbon ion radiotherapy and stereotactic body radiotherapy in stage I lung cancer. Anticancer Res 34: 5099-5104, 2014.

13 Kubo N, Saitoh JI, Shimada H, Shirai K, Kawamura H, Ohno T and Nakano T: Dosimetric comparison of carbon ion and X-ray radiotherapy for Stage IIIA non-small cell lung cancer. J Radiat Res 57: 548-554, 2016.

14 Kamada T, Tsujii H, Blakely EA, Debus J, De Neve W, Durante M, Jäkel O, Mayer R, Orecchia R, Pötter R, Vatnitsky S and Chu WT: Carbon ion radiotherapy in Japan: an assessment of 20 years of clinical experience. Lancet Oncol 16: e93-e100, 2015.

15 Mizoe JE, Hasegawa A, Jingu K, Takagi R, Bessyo H, Morikawa T, Tonoki M, Tsuji H, Kamada T, Tsujii H, Okamoto Y and Organizing Committee for the Working Group for Head Neck Cancer: Results of carbon ion radiotherapy for head and neck cancer, Radiother Oncol 103: 32-37, 2012.

16 Therasse P, Arbuck SG, Eisenhauer EA, Wanders J, Kaplan RS, Rubinstein L, Verweij J, Van Glabbeke M, van Oosterom AT, Christian MC and Gwyther SG: New guidelines to evaluate the response to treatment in solid tumors. J Natl Cancer Inst 92: 205-216, 2000 
17 Bradley JD, Paulus R, Komaki R, Masters G, Blumenschein G Schild S, Bogart J, Hu C, Forster K, Magliocco A, Kavadi V, Garces YI, Narayan S, Iyengar P, Robinson C, Wynn RB, Koprowski C, Meng J, Beitler J, Gaur R, Curran W Jr. and Choy $\mathrm{H}$ : Standard-dose versus high-dose conformal radiotherapy with concurrent and consolidation carboplatin plus paclitaxel with or without cetuximab for patients with stage IIIA or IIIB non-smallcell lung cancer (RTOG 0617): a randomized, two-by-two factorial phase 3 study. Lancet Oncol 16: 187-199, 2015.

18 Takahashi W, Nakajima M, Yamamoto N, Yamashita H, Nakagawa K, Miyamoto T, Tsuji H, Kamada T and Fujisawa T: A prospective nonrandomized phase I/II study of carbon ion radiotherapy in a favorable subset of locally advanced non-small cell lung cancer (NSCLC). Cancer 121(8): 1321-1327, 2015.

19 Ohno T, Oshiro Y, Mizumoto M, Numajiri H, Ishikawa H, Okumura T, Terunuma T, Sakae T and Sakurai H: Comparison of dose-volume histograms between proton beam and X-ray conformal radiotherapy for locally advanced non-small-cell lung cancer. J Radiat Res 56: 128-133, 2015.

20 Nichols RC, Huh SN, Henderson RH, Mendenhall NP, Flampouri S, Li Z, D'Agostino HJ, Cury JD, Pham DC and Hoppe BS: Proton radiation therapy offers reduced normal lung and bone marrow exposure for patients receiving dose-escalated radiation therapy for unresectable stage III non-small-cell lung cancer: a dosimetric study. Clin Lung Cancer 12: 252-257, 2011.

21 Oshiro Y, Okumura T, Kurishima K, Homma S, Mizumoto M, Ishikawa H, Onizuka M, Sakai M, Goto Y, Hizawa N, Sato Y and Sakurai H: High-dose concurrent chemo-proton therapy for Stage III NSCLC: preliminary results of a Phase II study. J Radiat Res 55: 959-965, 2014.
22 Harada H, Fuji H, Ono A, Kenmotsu H, Naito T, Yamashita H, Asakura H, Nishimura T, Takahashi T and Murayama S: Dose escalation study of proton beam therapy with concurrent chemotherapy for stage III non-small cell lung cancer. Cancer Sci 107: 1018-1021, 2016.

23 Lee H, Jeong SH, Jeong BH, Park HY, Lee KJ, Um SW, Kwon $\mathrm{OJ}$ and Kim H: Incidence of brain metastasis at the initial diagnosis of lung squamous cell carcinoma on the basis of stage, excluding brain metastasis. J Thorac Oncol 11: 426-431, 2016.

24 Ji Z, Bi N, Wang J, Hui Z, Xiao Z, Feng Q, Zhou Z, Chen D, Lv J, Liang J, Fan C, Liu L and Wang L: Risk factors for brain metastases in locally advanced non-small cell lung cancer with definitive chest radiation. Int J Radiat Oncol Biol Phys 89: 330337, 2014.

25 Noh JM, Kim JM, Ahn YC, Pyo H, Kim B, Oh D, Ju SG, Kim JS, Shin JS, Hong CS, Park H and Lee E: Effect of radiation therapy techniques on outcome in N3-positive IIIB non-small cell lung cancer treated with concurrent chemoradiotherapy. Cancer Res Treat 48: 106-114, 2016.
Received November 9, 2017

Revised November 20, 2017

Accepted November 23, 2017 